\title{
Confined magnetic guiding orbit states
}

\author{
J. Reijniers, A. Matulis 1 K. Chang \\ Departement Natuurkunde, Universiteit Antwerpen (UIA), \\ Universiteitsplein 1, B-2610 Antwerpen, Belgium \\ P. Vasilopoulos \\ Department of Physics, Concordia University, \\ 1455 de Maisonneuve Ouest, Montréal, Québec Canada, H3G1M8
}

\begin{abstract}
We show how snake-orbit states which run along a magnetic edge can be confined electrically. We consider a two-dimensional electron gas (2DEG) confined into a quantum wire, subjected to a strong perpendicular and steplike magnetic field $B /-B$. Close to this magnetic step new, spatially confined bound states arise as a result of the lateral confinement and the magnetic field step. The number of states, with energy below the first Landau level, increases as $B$ becomes stronger or as the wire width becomes larger. These bound states can be understood as an interference between two counter-propagating one-dimensional snake-orbit states.
\end{abstract}

PACS 73.20.-r, 73.23.-b, 73.40.-c

Recently there has been a growing interest in the study of the influence of inhomogeneous magnetic fields on the electronic properties of quantum structures. The application of magnetic fields to otherwise pure electrical systems opened the door to a whole new area of physics. The confining role of the magnetic fields can indeed alter the transport properties of, e.g., a two-dimensional electron gas (2DEG) to a measurable extent and therefore possibly result in useful magnetoelectronic devices. [1]

With regard to localized electron states, the confinement was previously realized essentially by application of pure electric potentials or pure inhomogeneous magnetic fields. However, the main problem with the latter method is of practical nature: it is very hard to create an inhomogeneous magnetic field which is strong enough to confine electrons such that the resulting energy quantization is large enough to detect. [2,3] In contrast to previous approaches, we propose a system which combines these two different ways of confinement, magnetic and electric, and which can be realized experimentally.

We consider the so-called snake-orbit states, which have been studied intensively in the recent past. [4 [6] These states are localized magnetically in one direction by use of large magnetic field gradients; a steplike magnetic field profile, which varies abruptly $B /-B$, e.g., along the $x$ direction, is a typical example, experimentally realizable. A typical feature of these states is that they propagate perpendicular to the magnetic field discontinuity (along the $y$ direction) and exhibit a large mobility. [5 As we show below, reducing this degree of freedom with hard-wall confinement leads to their complete spatial confinement.

To start with, we consider electrons in the $(x, y)$ plane subjected to a perpendicular magnetic field $\mathbf{B}=$ $[0,0, B(x)]$ which changes sign at $x=0$ such that $B(x, y)=B \operatorname{sgn}(x)$. Along the $y$ direction the electrons are confined into a quantum wire by a hard-wall poten- tial $V(|y|>W)=\infty$, else $V(y)=0$, where $W$ is the width of the wire. We solve the two-dimensional (2D) stationary Schrödinger equation

$$
\{H-E\} \Psi(x, y)=0
$$

with the dimensionless Hamiltonian

$$
H=-\left[\partial^{2} / \partial x^{2}+(\partial / \partial y+i|x| B)^{2}\right] / 2+V(y),
$$

where $|x| B$ is the $y$-component of the vector potential $\mathbf{A}$. The coordinates are measured in units of $W$, energy is in units of $E_{0}=\hbar^{2} / m_{e} W^{2}$, the magnetic field in $B_{0}=$ $\hbar / e W^{2} \cdot l_{B}=\sqrt{\hbar / e B}=W \sqrt{B_{0} / B}$ is the magnetic field length. For a typical GaAs-heterostructure with $W=$ $5000 \AA$, we obtain $E_{0} \approx 0.0056 \mathrm{meV}$ and $B_{0} \approx 26.4$ Gauss. The magnetic length reads $l_{B} \approx 0.045 W=224$ $\AA$.

This quantum mechanical problem cannot be solved analytically. One way to solve it is by using the modematching technique as in Ref. [7]. In the present work we follow a different approach and expand the wavefunction in basis functions of a $2 \mathrm{D}$ box in the absence of a magnetic field with boundaries at $|x|=L / 2,|y|=W / 2$, and the length of the wire $L$ chosen sufficiently large such that it has no influence on the energy of the bound state. Thus,

$$
\Psi(x, y)=\sum_{n=1}^{\infty} \sum_{m=1}^{\infty} c_{n m} \phi_{n m}(x, y),
$$

is the general form of the wave function and

$$
\phi_{n m}(x, y)=\frac{2}{\sqrt{W L}} \sin \left[\frac{n \pi}{L}\left(x+\frac{L}{2}\right)\right]\left[\frac{m \pi}{W}\left(y+\frac{W}{2}\right)\right]
$$

is the basis function of order $n, m$. Inserting Eq. (3) into Eq. (1) we obtain the secular equation

$$
\left|H_{i j}-E \delta_{i j}\right|=0
$$


where $H_{i j}=\left\langle\Psi_{i}|H| \Psi_{j}\right\rangle$ is the complex matrix element of the Hamiltonian operator with $i=(n, m)$. The energy of the bound state is then determined by solving Eq. (5). In principle an infinite number of basis functions is required to describe the wavefunction and hence to calculate the exact energy; in practice, though, we found that $n, m \approx 50$ terms are sufficient in order to determine the energy with an accuracy of $10^{-4}$.

If $B$ is larger than zero, an electron can be bound to the magnetic edge. We look at relatively large magnetic fields since we are interested in snake-orbit states, which are rather fragile structures and show themselves only for weak confining potentials. That is why we consider the case when the width of the wire $W$ exceeds the magnetic length $l_{B}$. The discrete energies in the box with dimensions $(L, W)$ subjected to a homogeneous magnetic field (dots) together with the steplike magnetic field profile (open dots) with strength $B / B_{0}=500$ are shown in Fig. 1. For the homogeneous magnetic field we clearly distinguish the first Landau level (solid dots). This level is also clearly seen for the $B /-B$ profile (open dots) and corresponds to electrons in cyclotron orbits on the left or right of the magnetic edge. But in addition, we now observe states with energy lower than the first Landau level. Inspection of the wavefunctions, plotted in the inset of Fig. 1 for $N=1$ and $N=5$, shows that the corresponding states are bound close to the magnetic boundary (situated at $x=0$ ).

The energy of the bound states, below the first Landau level, is shown in Fig. 2 as a function of the magnetic field strength $B / B_{0}=W^{2} / l_{B}^{2}$ in units of $\hbar \omega_{c}$, where $\omega_{c}=e B / m_{e}$ is the cyclotron frequency. We notice that with increasing $B$ the ground state decreases in energy and approaches the limiting value $E / \hbar \omega_{c}=0.295$ for $B \rightarrow \infty$. As $B$ increases the number of these bound states increases.

Actually these new bound states can be interpreted as an interference between two states propagating along the magnetic edge, i.e., perpendicular to the wire. These states have the same energy, lower than the first Landau level, but are propagating in opposite directions with different momenta, and for some energies they form a standing wave.

This can be better understood if we first look at the situation without confinement along $y$ described by $V(y)=0$, that was discussed in Ref. [4]. In this case one has to solve Eq. (11) with $W \rightarrow \infty$; now all lengths are expressed in units of $l_{B}$ and the energy in units of $\hbar \omega_{c}$. Writing the wave function as

$$
\psi(x, y)=e^{-i k y} \phi_{n, k}(x) / \sqrt{2 \pi},
$$

where $k$ is the wave vector in the $y$ direction, the problem reduces to solving the 1D Schrödinger equation

$$
(1 / 2)\left[-d^{2} / d x^{2}+(|x| B-k)^{2}\right] \phi_{n, k}(x)=E_{n, k} \phi_{n, k}(x),
$$

which we do numerically.
The energy spectrum, plotted in Fig. 3 as a function of $k$, shows that to every energy $E \leq \hbar \omega_{c} / 2$ there correspond two states which propagate along the magnetic boundary with opposite velocities $v_{y}\left(v_{y}=-\partial E / \partial k\right)$. The $v_{y}>0$ states are the classical snake-orbit states while those with $v_{y}<0$ do not have any classical analog.

In order to understand the nature of these different states, we distinguish two different regions, A and B. In region $\mathrm{B}$ the electron cyclotron orbit intersects the magnetic boundary and the electron motion can be understood classically. In region A there is no classical analog for the quantum mechanical electron propagation: classically, the orbit does not reach the magnetic boundary and the electron cycles around in closed orbits.

Since the lowest energy is at the border between the classical and non-classical region, only one of the two states with the same energy $E<\hbar \omega_{c}$ can be understood classically, the other cannot. Nevertheless, one could comprehend propagation of this state more or less, by looking at Fig. 目, where a schematic picture is drawn, together with the corresponding wavefunctions and their effective potential. If one adds tunneling to the classical picture, such that an electron can tunnel from a cycling state on the left to a cycling state on the right, and vice versa, one can imagine an electron to propagate parallel to the magnetic edge in the opposite direction of the classical snake-orbit [Fig. A(b)].

Now the question arises how this picture is modified if one includes a hard-wall confinement in the $y$-direction $V(y>W / 2)=\infty$. As argued below, the situation will change dramatically since the system is no longer translationally invariant along the $y$ direction and the wave function vanishes at the wire boundaries. The effect of the weak confinement can be taken into account qualitatively following the ideas used in band theory and assuming that the edge mode behaves like a quasi-particle moving along the magnetic well with effective mass $m^{*}=1.43 m_{e}$. Namely, expanding the electron energy near the minimum point $E_{0}$ for $k_{0} l_{B}=-0.767$ in the manner

$$
E(k) \rightarrow E_{0}+\left(k-k_{0}\right)^{2} / 2 m^{*},
$$

and presenting the electron wave function as

$$
\Psi(x, y)=f(y) \phi_{0}(x)
$$

[where $\phi_{0}(x)$ is the electron wave function at the minimum point $k_{0}$ ] one can get the effective Schrödinger equation for the envelope function $f(y)$. The Hamiltonian for this equation is obtained just replacing $k-k_{0} \rightarrow-i \partial / \partial y$ in energy expression (8) and adding the confinement potential:

$$
H_{0}=E_{0}-\left(1 / 2 m^{*}\right) \partial^{2} / \partial y^{2}+V(y) .
$$

In the case of hard wall confinement it immediately leads to the quantized energy spectrum

$$
E_{n}=E_{0}+\left(n \pi / l_{B}\right)^{2} / 2 m^{*},
$$


which is both qualitatively and quantitatively in good agreement with the exact result shown in Fig. 2, at least for lower energy values $\left(\left|k-k_{0}\right|\right.$ small). It is obvious that for higher $\left|k-k_{0}\right|$ the parabolic approximation (8) is not valid anymore.

The system studied here can be realized experimentally by growing a tilted 2DEG with the left and right parts having different slopes. [8] This results in an out-of-plane kink in the $1 \mathrm{D}$ wire. If a (large) homogeneous magnetic field is then applied, electrons will be attracted towards the kink where the magnetic field changes abruptly and form a quasi 1D system, which is confined both electrically and magnetically. Another way to create the steplike magnetic field in the wire is by growing a perpendicularly magnetized magnetic stripe on top of the heterostructure, containing the wire. [5] The magnetic field then has a different sign underneath or away from the stripe.

In summary, we proposed a technique to confine snakeorbit states and localize them spatially near a magneticfield discontinuity (or edge) by introducing an interference between them. These caged states are bound to the magnetic edge and have an energy lower than that of the first Landau level. Their number increases as the magnetic field becomes stronger or as the width of the wire becomes larger and the confinement decreases. These states can be understood as an interference between two counter-propagating snake-orbit states. We also provided a simple approximation which rendered clear their physical origin. They have a pure quantum mechanical origin and cannot be understood or treated classically.

This work was partially supported by the Interuniversity Micro-Electronics Center (IMEC, Leuven), the Flemish Science Foundation (FWO-Vl), the Belgian Interuniversity Attraction Poles (IUAP), and the FlemishChinese bilateral programme. J.R. was supported by "het Vlaams Instituut voor de bevordering van het Wetenschappelijk \& Technologisch Onderzoek in de Industrie" (IWT).

* Electronic mail: peeters@uia.ua.ac.be

- Electronic mail: amatulis@takas.lt

$\dagger$ Present Address: NLSM, Institute of Semiconductors, Chinese Academy of Sciences, Beijing 100089, China

[1] F. M. Peeters and J. De Boeck, in "Handbook of nanostructured materials and technology", Edited by N. S. Nalwa, Vol. 3 (Academic Press, N. Y., 1999), p. 345.

[2] J. Reijniers, A. Matulis and F. M. Peeters, Phys. Rev. B 64 (2001).

[3] N. Kim, G. Ihm, H. S. Sim, and T. W. Kang, Phys. Rev. B. 63, 235317 (2001).

[4] J. Reijniers and F. M. Peeters, J. Phys.: Condens. Matter 12, 9771 (2000).
[5] A. Nogaret, S. J. Bending and M. Henini, Phys. Rev. Lett. 84, 2231 (2000).

[6] J. Reijniers and F. M. Peeters, Phys. Rev. B 63, 165317 (2001).

[7] P. N. Butcher and J. A. McInnes, J. Phys.: Condens. Matter 7, 745 (1995).

[8] M. L. Leadbeater, C. L. Foden, T. M. Burke, J. H. Burroughes, M. P. Grimshaw, D. A. Ritchie, L. L. Wang, and M. Pepper, J. Phys.: Condens. Matter 7, L307 (1995).

FIG. 1. The lowest energy values for a quantum wire of width $W$ subjected to a homogeneous magnetic field $B / B_{0}=500$ (solid dots), and to a $B /-B$ profile (open dots). The inset shows the wave functions for the ground state $(N=1)$ and for the fifth lowest state $(N=5)$ in case of the $B /-B$ profile.

FIG. 2. The energies for the lowest bound states as function of the magnetic field strength $B$. The dotted lines correspond to our approximation [Eq. (11)], which is only shown for $E / \hbar \omega_{c}<0.35$. The thin dashed line corresponds to the lowest energy snake-orbit state in the absence of confinement.

FIG. 3. Dispersion relation of electrons subjected to a $B /-B$ profile. The thin solid line separates the different regions $\mathrm{A}$ and $\mathrm{B}$ corresponding, respectively, to states which cannot and can be understood classically. The bound states with energy below the first Landau state are represented by the bold line. The dotted line shows the displaced parabolic approximation with $k_{0} / l_{B}=-0.767, E_{0} / \hbar \omega_{c}=0.295$, $m^{*} / m_{e}=1.43$.

FIG. 4. Schematic trajectories and corresponding wave functions for the states indicated by the solid dots in Fig. B, i.e. for $k l_{B}=-0.3$ and for $k l_{B}=-1.35$. 
This figure "fig1.jpg" is available in "jpg" format from: http://arxiv.org/ps/cond-mat/0112155v1 
This figure "fig2.jpg" is available in "jpg" format from: http://arxiv.org/ps/cond-mat/0112155v1 
This figure "fig3.jpg" is available in "jpg" format from: http://arxiv.org/ps/cond-mat/0112155v1 
This figure "fig4.jpg" is available in "jpg" format from: http://arxiv.org/ps/cond-mat/0112155v1 